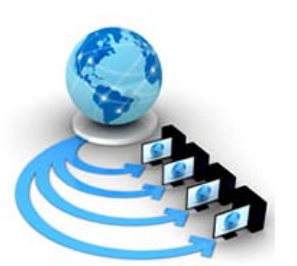

\title{
EFFICIENTLY RECOGNIZING THE FACE USING HYBRID APPROACH OF GAUSSIAN FILTERING AND SIFT KEY ALGORITHM
}

\author{
Sapna Bharti \\ M.tech Scholar \\ SSIET, Dinanagar,India
}

\author{
Bhavna Saini \\ Assistant Professor \\ SSIET, Dinanagar,India
}

\begin{abstract}
Automatic Face Recognition System becomes the challenging problem due to its requirement in various applications like defense, surveillance and access control.It helps to identify the face image correctly and store them in database. In real time applications appearance of face changes due to different pose, expression, affine transformations and illumination etc. In our research paper to obtain accurate results and good performance Modified SIFT (Scale Invariant Feature Transform) technique is used. By using modified SIFT approach difference between original and query database is identified. The proposed approach is efficient in performance and provides experimental results on the basis of developed algorithm for accurately recognizing the images from dataset.
\end{abstract}

Keywords- Face Recognition, SIFT, noise, HSIFT

\section{INTRODUCTION}

Because of expanding security requests, its potential business or law authorization applications confront acknowledgment has turned into an extremely dynamic territory of research lately. The acknowledgment procedure of this framework incorporated the area of components like eyebrows, eyes, noses, computation of separations and proportions to a typical reference point and layout coordinating.[2]Despite the fact that there are various face acknowledgment calculations which function correctly in obliged conditions, confront acknowledgment is as yet an open and extremely difficult issue in genuine applications. Among oppose acknowledgment calculations, appearancebased methodologies are the most prominent. These methodologies use the pixel force or power inferred highlights. A few such frameworks have been effectively created and introduced. Be that as it may, appearance-based techniques don't perform well in some true circumstances, where the inquiry test confront appearance is fundamentally unique in relation to the preparation confront information, because of varieties in posture, lighting and articulation.

It is an appealing component of the front line human-PC interfaces. PCs that can see outward appearances and respond to the sentiments of individuals properly enable better human machine correspondence. Appearance of the face relies upon the geometry, surface, shape and development of facial components.

Face Recognition winds up plainly difficult task if there should arise an occurrence of changing outward appearances. In such case focal inclination are required to be utilized as a part of request to have a main issue speaking to regular face behavior out of huge number of appearances. This point help in recognizable proof of face when looked at against preparing set of pictures. The face looks of a man are proficient medium to pass on points, emotions and the internal viewpoint. One of the issues that any facial acknowledgment framework must be fit to manage various pictures of a comparative face pictures which have distinctive size, position, lightning conditions, and outward appearance. Thus it may be hard to consider different pictures of a comparable face under different conditions. Facial acknowledgment frameworks tend to fuse a predealing with organize where the picture is institutionalized concerning the beforehand specified combination i.e. the picture is changed to some standard size and position etc.

Size and position are by and large easy to oversee yet the others are troublesome.

Face acknowledgment gives the forefront progresses in business law authorization and military applications. It has furthermore drawn huge interest and thought from various experts all through the past two decades because of its potential applications in the regions of observation and customer approval. The face is a champion among the most attractive biometrics, and it has in like manner been the most essential method for recognition that human use in their visual associations. The issue with confirmation systems in light of unique mark, voice, iris and the present quality structure (DNA unique mark) has been the issue of data obtainment or procurement.

For unique finger impression the concerned individual should keep his/her finger in fitting position and in case of speaker recognition the beneficiary should be kept in genuine position and partition from the speaker. Regardless, the technique for obtaining face pictures is nonintrusive and the face can be used as a biometric property.

[3](Mukhedkar 2015)Face is a comprehensive part of individuals. Face recognition is basic not in view of the capacity of its piece of potential applications in investigate fields also in light of the limit of its answer which would help in settling other portrayal issues like question recognition. 


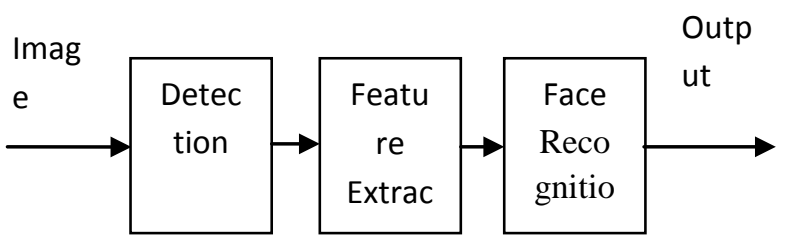

Figure 1: Face Detection Process

In some satisfying frameworks, face detection is preventing by obliging the client. Most frameworks utilize a mix of skin-tone and face surface to decide the area of a face and utilize a picture pyramid to enable faces of differing sizes to be distinguished.[4] Progressively, frameworks are being produced to identify faces that are not full-frontal. Prompts, for example, development and individual detection can be utilized to limit faces for recognition. Commonly interpretation, scale and in-plane turn for the face are assessed at the same time, alongside pivot inside and out when this is considered.

\section{FACE RECOGNITION}

There is an extraordinary presentable variety in the way facial appearance is translated for recognition by a programmed framework. At present various distinctive frameworks are being worked on, and which is most fitting may rely upon the application area. A noteworthy contrast in approaches is whether to speak to the presence of the face, or the geometry. I have looked at the two methodologies; in any case most frameworks today utilize a mix of both appearance and geometry. Geometry is hard to measure with any exactness, especially from a solitary still picture, however gives more power against masks and maturing. Appearance data is promptly acquired from a face picture yet is more subject to superial variety, especially from posture and behavior changes. By and by for most purposes, even appearance-based frameworks must measure some geometrical parameters with a specific end goal to determine a 'shape free' portrayal that is autonomous of articulation and stance relics. [5]

This is accomplished bfynding facial land stamps and twisting the face to an authoritative unbiased posture and appearance. Facial features are likewise critical for geometric methodologies and for tying down neighborhood portrayals. Face appearance portrayal plans can be isolated into neighborhood and worldwide, contingent upon whether the face is spoken to all in all, or as a progression of little locales. Most worldwide methodologies depend on a primary parts portrayal of the face picture powers. This portrayal conspire was conceivfidst for face picture pressure purposes and along these lines utilized for recognition purposes. The last authored the term Eigen faces for this sort of portrayal. A face picture is spoken to as a vector of forces and this vector is then approximated as an entirety of premise vectors (Eigen faces) figured by key part investigation from a database of face pictures. These primary segments speak to the regular varieties seen amongst faces and give a brief epitome of the presence of a specimen face picture, and a reason for its examination with other face pictures. This important segments portrayal is, as for instance the Fourier Transform, a de connecting change to an option premise where great portrayals of the remarkable attributes of a picture can be made from just a couple of low-arrange coefficients in spite of disposing of a hefty portion of the higher-arrange terms[15].

\section{FEATURE EXTRACTION/SELECTION}

Feature extraction includes a few stages - dimensionality lessening, feature extraction and feature choice. Dimensionality lessening is a fundamental undertaking in any example recognition framework. The execution of a classifier relies upon the measure of test pictures, number of features and classifi er multifaceted nature. This prerequisite ought to be satfied when constructing a classifier. The more perplexing the classier, the bigger ought to be the said proportion. This "reprove" is one reason why it's critical to keep the quantity of features as little as could be expected under the circumstances. The other principle reason is the speed. The classfier will be speedier and will utilize less memory. Additionally, a substantial arrangement of features can bring about a false positive when these features are excess. Eventually, the quantity of features must be precisely picked. Too less or repetitive features can prompt lost exactness of the recognition framework.

A feature extraction calculation removes features from the information. It makes those new features in light of changes or mixes of the first information. A feature determination calculation chooses the best subset of the information feature set. It disposes of non-applicable features. Feature determination is frequently performed after feature extraction. In this way, features are separated from the face pictures, at that point an ideal subset of these features is chosen. The dimensionality decrease process can be implanted in some of these means, or performed before them.

Face recognition is utilized to perform two principle essential tasks i.e. check and ID. Verification is the way toward confirming a man to perceive an obscure individual alongside a claim of personality and to guarantee whether the individual is same is called verification. Recognizable proof is a procedure to distinguish a man by looking at his/her face with the given database [1]. Face recognition frameworks are for the most part used to recognize people, for get to control, for security purposes and there is numerous other utilization of face recognition framework, a couple of which are given underneath:

-Security of structures, air terminals, seaports, programmed teller machines (ATM) machines and fringe checkpoints.

-Used in different territories of teach for contrast a substance and an arrangement of element.

-For Surveillance through CCTVs to search for known culprits

- General character confirmation at banks, organizations and different associations.

-Examination for improper records.

-Significant in procedure of some VIP is coming in the inn. - Criminal equity frameworks through post-occasion investigation and legal applications.

-Investigations of picture database like looking of picture databases like authorized drivers advantage beneficiaries, missing kids, migrants and so on. 
-In brilliant card applications where the face picture can be put away in a keen card, scanner tag or attractive stripe, to verify the live picture and the put away picture.

-Labeling the face pictures in video called video Indexing.

-For the order of sex.

-In numerous restorative field like psychiatry for stress and discouragement discovery.

Any undesirable thing in a picture is considered as a commotion. Since the execution of any face recognition system relies upon the nature of caught picture of the face to be perceived. The de-noising calculation expels the noise part while safeguarding the first picture structures. In the vast majority of the applications, the nearness of commotion in picture prompts awful execution of consequent picture preparing undertakings which are entirely subject to the achievement of de-noising operation. The issue in face recognition framework is the recognition of loud face picture. The recognition of loud picture is exceptionally troublesome task and this may come about into wrong recognition. Then again, it is important to guarantee that denoising system must not twist the valuable data in the picture and should protect picture subtle elements and surface while expelling the noise. It is exceptionally important to expel commotion in the pictures previously applying any further picture preparing assignment, for example, picture recognition, edge location, picture division and so on.

The commotion may enter in a picture as a result of camera movement, poor camera sensors, environmental conditions or boisterous transmission medium. Electronic commotion related with a capacity recovery framework can likewise be the reason of debasement of picture. It is an extremely normal issue. A picture can likewise get defiled amid the securing forms, or while its stockpiling and recovery.

There are diverse sorts of noise. Commotion might be sorted into substitutive noise e.g., salt and pepper noise, irregular esteemed motivation noise, and so forth and added substance noise e.g., added substance white gaussiannoise. The substitutive commotion of low and normal noise densities can be effortlessly evacuated with the assistance of de-noising plans accessible in the writing. The middle channel performs exceptionally well to remove drive commotion at low thickness. There are numerous other denoising plans are proposed which are well skilled in expelling motivation noise at low to high commotion densities. Then again added substance commotion is extremely hard to expel on the grounds that it debases every one of the pixels display in the picture. The Mean channel can be connected to expel added substance noise however it additionally obscure the picture while evacuating commotion.

\section{LITERATURE SURVEY}

[6] (Murtaza et al. 2013) presented an entire review of face recognition led under shifting outward appearances. In order to examine distinctive systems, movements based, demonstrate based and muscles-based methodologies have been utilized as a piece of demand to deal with the outward appearance and recognition calamity. The investigation has been finished by assessing different existing calculations while looking at their results when in doubt. It additionally extends the extension for different scientists and effectively managing such issues.

[7] (Liu et al. 2014) proposed that idealistic faces created better personality recognition concerning shake faces, paying little regard to whether they were tried in a comparative picture or another photo showing a neutral attitude. None of the other passionate expressions made quantifiable preferred standpoint for recognition memory. Notwithstanding, the point by point investigations additionally demonstrate that the upside of glad expression on character recognition may not be comparably recognizable from all other enthusiastic expressions.

[8] (Ma n.d.) Proposed technique increases the BU-4DFE dataset by adding distinctive lighting conditions to $3 \mathrm{D}$ pictures of subjects performing diverse facial appearances. They build up a photo handling pipeline to amend the effects of brightening on the photos, wanting to secure high arrangement rate even in brutal lighting conditions. By then they test the pipeline on two estimations: grouping precision in perspective of a LDA model and SIFT key point repeatability. The photo handling pipeline enhanced order precision when performing LDA to recognize pictures in dim lighting conditions. They didn't discover noteworthy change in key point discovery.

[9](Anon n.d.) extended the prevalence of Face Recognition (FR) systems have expanded in light of their utilization in boundless applications, for instance, biometric (distinguishing evidence and validation), security (Banks, air terminals, and so on.) and surveillance (missing youngsters or finding outlaw crooks) structures, and in addition picture and video ordering systems. FR has been a solid field of research since the 1990s, however still a long route from is dependable and more procedures are being developed each year. FR investigate region primary challenges are, a couple of individuals faces recognition won't not fill in and in addition for others (for instance, long hair or facial hair, feelings, lighting, and foundation may give additional inconvenience). Most by far of the exploration specialists solidly assume that feelings of a man expect the critical part in essential leadership.

[10] (Neeru and Kaur 2016a) provided the recognition of a face under different feelings is a testing subject. The work done in this paper is twofold. In any case, Local binary Pattern (LBP) and center symmetric Local binary Pattern (CS-LBP) has been connected to separate the nearby parallel components of the photo. Second, Euclidean distance, histogram convergence and chi-square separation is utilized for recognition of face. The execution is assessed on the Japanese Female Facial Expression (JAFFE) database and results are analyzed the extent that recognition rate and time taken for handling. It has been watched that CS-LBP gives best recognition rate rather finished LBP in the event of different appearances of face.

[11] (Neeru and Kaur 2016b) build up a completely programmed face recognition calculation. Scale Invariant Feature Transform (SIFT) has effortlessly been utilized as a piece of face recognition. In this paper, a Modified SIFT (MSIFT) approach has been proposed to enhance the recognition execution of SIFT. In this paper, the work is done in three phases. To begin with, the smoothing of the photo has been finished utilizing DWT. Second, the computational multifaceted nature of SIFT in descriptor figuring is diminished by subtracting normal from each 
descriptor instead of standardization. Third, the calculation is made programmed by utilizing Coefficient of Correlation (CoC) as opposed to utilizing the separation proportion (which requires client communication).

[11] (Chennamma and Rangarajan 2010) proposed another approach for face unmistakable evidence from controlled facial pictures in light of SIFT parts. The proposed approach is differentiated and Eigen faces and showed its prevalence through trials. In this paper, they concentrated just on mug shot recognizable proof. As an expansion, they inspected the usage of SIFT components for recuperation of right face with various sorts of face delegates.

[12] (Seo and Park 2014) took care of 2D pictures be removing segments of the photo. Features extraction instrument uses SIFT (Scale invariant Feature change). Channel is used to address neighborhood incorporates as a descriptor. Variety in facial pictures is perceived by the use of probability thickness work found out from SIFT. Benchmark datasets are used for distinguishing proof of facial varieties through proposed method.

[13] (Gupta and Garg 2014) SIFT used for face recognition on different invariants and accuracy was figured. Close examination among various invariants was moreover a bit of this paper by giving the two figures and estimations. Through the examination it is demonstrated that they deduce that SIFT works amazingly well for articulation and camera determination. It gives normal results when pictures are of different lighting up levels. Channel is fitting to check pictures having about same edification, with different position, articulation, embellishments etc..

[14] (Mohanraj et al. 2016) proposed Hybrid SIFT plan to see face in video under different edification and position variety. The proposed system uses light modification channel of Retina Modeling for pre-taking care of and Histogram of Oriented Gradients for face area, as it has lesser false positive rates. Remembering the true objective to beat the lighting up issue, the main SIFT is changed in this paper with settled point of reference localizer to stamp the key concentrations from which the presentation task of SIFT is done and a light invariant part vector is gotten.

[16] (Fallis 13AD) depicted that Individuals are normally recognized by their faces. Headways in the past couple of decades have engaged human to thusly do the conspicuous evidence process. Early face recognition calculation used fundamental geometric models. Directly, face recognition get ready uses the advanced factual science and organizing procedures. Changes and improvements in face recognition development in the midst of 10 to 15 past years have pushed it to the present status. Face recognition is applicable for both examination and ID. This audit gave another face recognition technique in light of SIFTS components. Upgrading SIFT calculation, this survey focused on face recognition. Comes to fruition demonstrated the prevalence of the proposed calculation over the SIFT. The results gotten from various tests exhibited that the proposed calculation, with $98.75 \%$ accuracy and run time of 4.3 seconds, is more capable and correct than various calculations yet the run time was shy of what others.

\section{OBJECTIVE}

Face detection is a system of recognizing particular outward appearance by the utilization of picture preparing procedures. The human face is dynamic having particular appearance at unmistakable time. The human face is 3D protest displaying distinctive outward appearance. Projection from $3 \mathrm{D}$ to $2 \mathrm{D}$ is basic in the examination procedure as a few subtle elements might be lost. So a few credits must be converged in existing ways to deal with improve face acknowledgment process. The proposed framework handles the outward appearance acknowledgment with ideal acknowledgment rate. The destinations are recorded as takes after

- $\quad$ Extracting the components from the preparation set utilizing adjusted SIFT keys with middle channel for effective extraction taking out low differentiation pixels..

- After extracting the components, performing determination of ideal elements related with significant appearance of face with scale invariant element choice.

- Enhancing precision of result created and thought about against other classifier.

- Enhancing execution of outward appearance detection and contrasting it and diverse methodologies as far as acknowledgment rate.

Proposed Methodology

The proposed work is described in this section. The proposed work takes the optimal properties of various algorithms along with genetic algorithm to produce optimal rate corresponding to face recognition.

DATASET COLLECTION

In order to prove worth of research corresponding to face recognition two datasets are used from the internet.

YALE DATASET: This dataset contains 15 image set of distinct Male individuals with 11 images per individual. Images are in GIF format. Gray scale images of individual are loaded within this training set.

NLPR DATASET: This dataset contains 450 Male and Female face images. The image format is JPEG. So conversion from JPEG to GIF is required. Each image has 896x592 pixels.

\section{FEATURE EXTRACTION}

This is a procedure by which features are separated from starting arrangement of preparing pictures. Dimensionality is incredibly decreased by the utilization of feature extraction. Basically excess data is wiped out thus speed of acknowledgment is significantly upgraded. For extricating the features SIFT (Scale Invariant Feature change) is utilized. Filter for the most part utilize Gaussian channel to choose potential key point yet in proposed work middle channel is utilized to choose key focuses. However every one of the features removed may not respect grouping comes about. Thus feature selection is required to be performed.

\section{FEATURE SELECTION}

Feature selection extraordinarily decreases dimensionality to upgrade rate of acknowledgment. However every one of the features separated may not add to arrangement comes about. Thus features that add to various significant classes feature 
selection is required to be performed. Features like mean, criticized deviation, Entropy, RMS, difference, smoothness, Kurtosis, IDM, Contrast, connection, Energy, Homogeneity are shaped particular classes. The features produced through extraction process checked against the class passage. On the off chance that feature don't exist in any class at that point feature is rejected. Consequently dimensionality is additionally lessened.

\section{Proposed Methodology}

Proposed methodology used hybrid approach of buffer mechanism along with HSIFT and RWBGA. The steps which are performed can be described as:

Apply Face Acquisition and selection procedure to select particular test image from Training set.

1. Apply modified median filter to reduce noise if any from the image

2. Test_Image $=$ median2(Test_Image $)$

3. After Applying Pre-processing, apply HSIFT for feature extraction

4. Apply genetic approach with roulette wheel selection for feature selection

5. Repeat the above listed steps until termination criteria is satisfied or optimal result is obtained

Feature extraction with HSIFT

Besides include Selection utilizing Modified SIFT key will be assessed. . Face acknowledgment is done by restricting the objective work which prompts assurance of perfect course of action of trusty centers or SIFT Points. The technique shields the close-by information from different facial points of view for mapping neighboring commitment to its looking at yield, achieving low dimensional depiction for encoding the associations of the data. The proposed procedures Hexagonal Descriptor Particle Swarm Optimization with Knowledge-Crowding (HDPSO-KC) overcomes from adjacent optima and upgrades overall chase plan and communitarian work. The system in like manner covers the issue of shedding the particles in denser zones in Pareto front scattering. (Chennamma and Rangarajan 2010)The proposed system is affirmed with benchmark datasets for examining the execution over various methods. Lowe's system for picture feature period changes a photo into an extensive social occasion of feature vectors, each of which is invariant to picture understanding, scaling, and turn, mostly invariant to lighting up changes and generous to adjacent geometric reshaping. These components share practically identical properties with neurons in basic Visual cortex that are encoding central structures, shading and advancement for question recognizable proof in primate vision. Key regions are portrayed as maxima and minima of the outcome of qualification of Gaussians limit associated in scale space to a movement of smoothed and resampled pictures. Low multifaceted nature candidate centers and edge response centers along an edge are discarded. Winning acquaintances are doled out with restricted key focuses. These methods ensure that the key focuses are all the more consistent to match and affirmation. Channel descriptors generous to neighborhood relative twisting are then gotten by considering pixels around a traverse of the key range, darkening and resampling of close-by picture presentation planes.
Feature selection with RWBGA

In third step extraction of face elements to arrange the picture utilizing Genetic calculation with roulette Wheel Selection. In this chromosome assurance handle occurs on the start of roulette wheel. Roulette wheel assurance should be possible by the crucial bit of the decision technique is to stochastic accomplice select from one time to make commence of the general population to come. The need is that the fittest individuals have a more conspicuous shot of survival than weaker ones. This reproduces nature in that fitter individuals will tend to have an unrivaled probability of survival and will proceed to outline the mating pool for the general population to come. Weaker individuals are not without plausibility. In nature such individuals may have innate coding that may exhibit supportive to who and what is to come. After that assurance of the fittest has been finished.

Flowchart

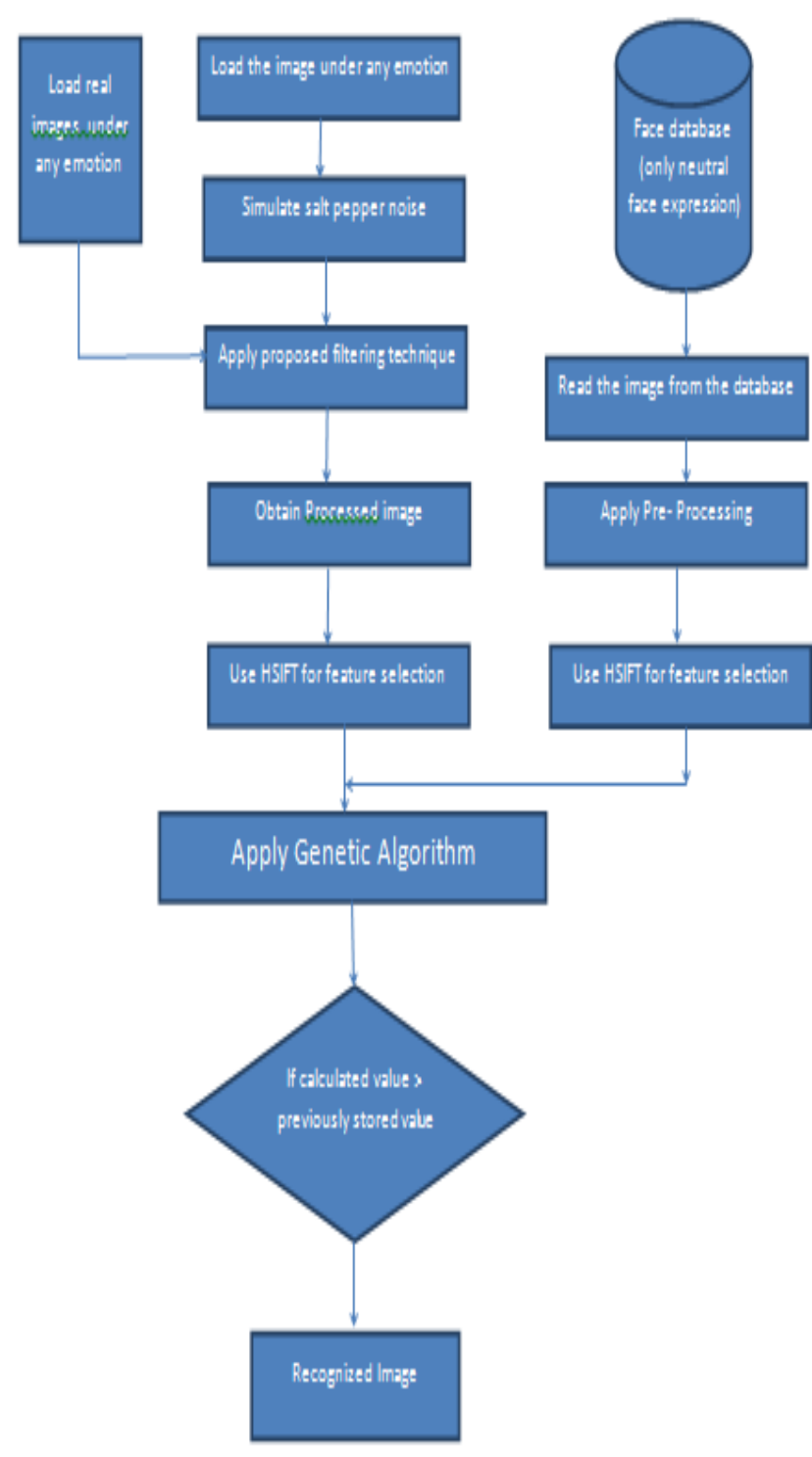

Results

\section{YALE DATASET RESULTS}

This dataset contains 15 image set of distinct Male individuals with 11 images per individual[17] .(Yang et al. 
2004) Images are in GIF format. Gray scale images of individual are loaded within this training set. The database contains 5760 single light source pictures of 10 subjects each observed under 576 review conditions ( 9 postures x 64 brightening conditions). [18] (Belhumeur et al. 1997) For each subject in a specific represent, a picture with encompassing (foundation) brightening was likewise caught. The following table shows the recognition rate of various training sets in YALE dataset using different techniques

Table 1: Comparison of results of various techniques with purposed methodology using YALE dataset

\begin{tabular}{|c|c|c|c|c|c|}
\hline $\begin{array}{l}\text { Traini } \\
\text { ng Set }\end{array}$ & $\begin{array}{l}\text { Propos } \\
\text { ed }\end{array}$ & PCA & DWT & $\begin{array}{l}\text { DWT+P } \\
\text { CA }\end{array}$ & $\begin{array}{l}\text { DWT+ } \\
\text { CC }\end{array}$ \\
\hline $\begin{array}{l}\text { Traini } \\
\text { ng } \\
\text { Set1 }\end{array}$ & $\begin{array}{l}97.608 \\
7\end{array}$ & $\begin{array}{l}89.78 \\
01\end{array}$ & $\begin{array}{l}93.05 \\
41\end{array}$ & 93.0401 & 89.8831 \\
\hline $\begin{array}{l}\text { Traini } \\
\text { ng } \\
\text { Set2 }\end{array}$ & $\begin{array}{l}94.022 \\
3\end{array}$ & $\begin{array}{l}93.20 \\
43\end{array}$ & $\begin{array}{l}94.68 \\
42\end{array}$ & 94.6662 & 93.1981 \\
\hline $\begin{array}{l}\text { Traini } \\
\text { ng } \\
\text { Set3 }\end{array}$ & $\begin{array}{l}73.065 \\
2\end{array}$ & $\begin{array}{l}55.92 \\
79\end{array}$ & $\begin{array}{l}64.24 \\
06\end{array}$ & 64.2447 & 57.6053 \\
\hline $\begin{array}{l}\text { Traini } \\
\text { ng } \\
\text { Set4 } \\
\end{array}$ & $\begin{array}{l}91.023 \\
2\end{array}$ & $\begin{array}{l}71.34 \\
55\end{array}$ & $\begin{array}{l}89.23 \\
43\end{array}$ & 87.0873 & 88.6089 \\
\hline $\begin{array}{l}\text { Traini } \\
\text { ng } \\
\text { Set5 }\end{array}$ & $\begin{array}{l}95.114 \\
3\end{array}$ & $\begin{array}{l}80.98 \\
50\end{array}$ & $\begin{array}{l}88.89 \\
0\end{array}$ & 85.8792 & 86.8769 \\
\hline $\begin{array}{l}\text { Traini } \\
\text { ng } \\
\text { Set6 }\end{array}$ & $\begin{array}{l}82.230 \\
4\end{array}$ & $\begin{array}{l}70.57 \\
44\end{array}$ & $\begin{array}{l}83.34 \\
24\end{array}$ & 81.0122 & 82.9024 \\
\hline $\begin{array}{l}\text { Traini } \\
\text { ng } \\
\text { Set7 }\end{array}$ & $\begin{array}{l}83.530 \\
4\end{array}$ & $\begin{array}{l}72.47 \\
59\end{array}$ & $\begin{array}{l}78.23 \\
44\end{array}$ & 75.8549 & 77.3624 \\
\hline $\begin{array}{l}\text { Traini } \\
\text { ng } \\
\text { Set8 } \\
\end{array}$ & $\begin{array}{l}80.145 \\
8\end{array}$ & $\begin{array}{l}71.59 \\
84\end{array}$ & $\begin{array}{l}79.25 \\
48\end{array}$ & 78.8451 & 79.5214 \\
\hline $\begin{array}{l}\text { Traini } \\
\text { ng } \\
\text { Set9 }\end{array}$ & $\begin{array}{l}85.245 \\
6\end{array}$ & $\begin{array}{l}72.54 \\
8\end{array}$ & $\begin{array}{l}84.25 \\
46\end{array}$ & 82.1245 & 85.3654 \\
\hline $\begin{array}{l}\text { Traini } \\
\text { ng } \\
\text { Set10 }\end{array}$ & $\begin{array}{l}90.254 \\
8\end{array}$ & $\begin{array}{l}80.26 \\
58\end{array}$ & $\begin{array}{l}88.36 \\
54\end{array}$ & 86.4578 & 87.3654 \\
\hline $\begin{array}{l}\text { Traini } \\
\text { ng } \\
\text { Set11 }\end{array}$ & $\begin{array}{l}75.595 \\
4\end{array}$ & $\begin{array}{l}63.98 \\
74\end{array}$ & $\begin{array}{l}70.58 \\
74\end{array}$ & 68.4825 & 70.2525 \\
\hline $\begin{array}{l}\text { Traini } \\
\text { ng } \\
\text { Set12 } \\
\end{array}$ & $\begin{array}{l}86.245 \\
7\end{array}$ & $\begin{array}{l}72.58 \\
44\end{array}$ & $\begin{array}{l}84.24 \\
98\end{array}$ & 81.9548 & 82.4579 \\
\hline $\begin{array}{l}\text { Traini } \\
\text { ng } \\
\text { Set13 }\end{array}$ & $\begin{array}{l}98.245 \\
8\end{array}$ & $\begin{array}{l}80.25 \\
48\end{array}$ & $\begin{array}{l}87.63 \\
25\end{array}$ & 85.6642 & 86.9548 \\
\hline $\begin{array}{l}\text { Traini } \\
\text { ng } \\
\text { Set14 }\end{array}$ & $\begin{array}{l}97.897 \\
4\end{array}$ & $\begin{array}{l}85.65 \\
48\end{array}$ & $\begin{array}{l}95.64 \\
87\end{array}$ & 93.1452 & 94.2455 \\
\hline $\begin{array}{l}\text { Traini } \\
\text { ng } \\
\text { Set15 }\end{array}$ & $\begin{array}{l}95.548 \\
7\end{array}$ & $\begin{array}{l}87.24 \\
58\end{array}$ & $\begin{array}{l}94.55 \\
78\end{array}$ & 92.3258 & 93.1424 \\
\hline $\begin{array}{l}\text { Traini } \\
\text { ng } \\
\text { Set16 }\end{array}$ & $\begin{array}{l}94.025 \\
4\end{array}$ & $\begin{array}{l}85.58 \\
75\end{array}$ & $\begin{array}{l}91.01 \\
45\end{array}$ & 90.2547 & 92.5874 \\
\hline
\end{tabular}

\begin{tabular}{|l|l|l|l|l|l|}
\hline $\begin{array}{l}\text { Traini } \\
\text { ng } \\
\text { Set17 }\end{array}$ & $\begin{array}{l}82.564 \\
8\end{array}$ & $\begin{array}{l}75.85 \\
48\end{array}$ & $\begin{array}{l}80.54 \\
84\end{array}$ & 78.6954 & 80.3254 \\
\hline $\begin{array}{l}\text { Traini } \\
\text { ng } \\
\text { Set18 }\end{array}$ & $\begin{array}{l}81.369 \\
5\end{array}$ & $\begin{array}{l}73.14 \\
58\end{array}$ & $\begin{array}{l}80.65 \\
98\end{array}$ & 78.9548 & 80.6985 \\
\hline $\begin{array}{l}\text { Traini } \\
\text { ng } \\
\text { Set19 }\end{array}$ & $\begin{array}{l}96.548 \\
\text { Traini }\end{array}$ & $\begin{array}{l}85.55 \\
66\end{array}$ & $\begin{array}{l}92.65 \\
87\end{array}$ & 90.8654 & 91.2354 \\
$\begin{array}{l}\text { ng } \\
\text { Set20 }\end{array}$ & 5 & $\begin{array}{l}86.58 \\
96\end{array}$ & $\begin{array}{l}92.47 \\
85\end{array}$ & 91.8569 & 92.7894 \\
\hline
\end{tabular}

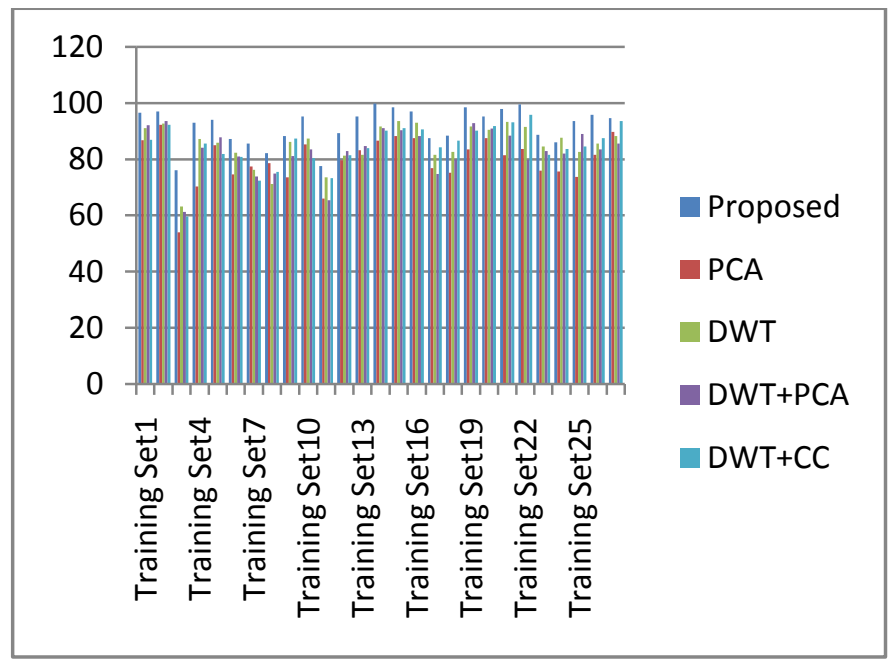

Figure 2: Comparison of results of various techniques with purposed methodology using YALE dataset

CONCLUSION AND FUTURE SCOPE

The proposed approach uses modified SIFT(involving median filter) with enhanced genetic algorithm involving roulette wheel selection model in order to detect the face effectively. Set of 11 distinct feature classes are used. Feature selection is used to reduce the search space further. Genetic algorithm is used in order to optimally select the feature. For this purpose roulette wheel selection is performed. Recognition rate and accuracy is significantly enhanced through proposed technique(SIFT+GA). In other words optimal features are selected after extraction and then evaluation is performed using GA. The proposed approach analyses the object from different viewpoints and then object matching highest key point is selected as a destination image. All the critical features are selected and hence discrimination ability is high. The feature selection is based on objective function value. Better convergence in terms of recognition rate is observed through proposed technique.

\section{REFERENCES}

1. Belhumeur, P.N., Hespanha, J.P. \& Kriegman, D.J., 1997. Eigenfaces vs. fisherfaces: Recognition using class specific linear projection. IEEE Transactions on Pattern Analysis and Machine Intelligence, 19(7), pp.711-720.

2. Chennamma, H.R. \& Rangarajan, L., 2010. Face Identification from Manipulated Facial Images Using SIFT. 2010 3rd International Conference on Emerging Trends in Engineering and Technology, pp.192-195. Available at: http://ieeexplore.ieee.org/lpdocs/epic03/wrapper.htm?arnum ber $=5698318$. 
3. Lu, X.L.X., Wang, Y.W.Y. \& Jain, A.K., 2003. Combining classifiers for face recognition. 2003 International Conference on Multimedia and Expo., 3, pp.13-16.

4. Mukhedkar, M.M., 2015. Fast Face Recognition Based on Wavelet Transform on PCA. , (Icesa), pp.761-764.

5. Tian, Y. et al., 2003. Do singular values contain adequate information for face recognition? Pattern Recognition, 36(3), pp.649-655.

6. Yang, J. et al., 2004. Two-Dimensional PCA: A New Approach to Appearance-Based Face Representation and Recognition. IEEE Transactions on Pattern Analysis and Machine Intelligence, 26(1), pp.131-137.

7. Belhumeur, P.N., Hespanha, J.P. \& Kriegman, D.J., 1997. Eigenfaces vs. fisherfaces: Recognition using class specific linear projection. IEEE Transactions on Pattern Analysis and Machine Intelligence, 19(7), pp.711-720.

8. Chennamma, H.R. \& Rangarajan, L., 2010. Face Identification from Manipulated Facial Images Using SIFT. 2010 3rd International Conference on Emerging Trends in Engineering and Technology, pp.192-195. Available at: http://ieeexplore.ieee.org/lpdocs/epic03/wrapper.htm?arnum ber $=5698318$.

9. Lu, X.L.X., Wang, Y.W.Y. \& Jain, A.K., 2003. Combining classifiers for face recognition. 2003 International Conference on Multimedia and Expo., 3, pp.13-16.

10. Mukhedkar, M.M., 2015. Fast Face Recognition Based on Wavelet Transform on PCA. , (Icesa), pp.761-764.

11. Tian, Y. et al., 2003. Do singular values contain adequate information for face recognition? Pattern Recognition, 36(3), pp.649-655.

12. Yang, J. et al., 2004. Two-Dimensional PCA: A New Approach to Appearance-Based Face Representation and Recognition. IEEE Transactions on Pattern Analysis and Machine Intelligence, 26(1), pp.131-137.

13. Belhumeur, P.N., Hespanha, J.P. \& Kriegman, D.J., 1997. Eigenfaces vs. fisherfaces: Recognition using class specific linear projection. IEEE Transactions on Pattern Analysis and Machine Intelligence, 19(7), pp.711-720.

14. Chennamma, H.R. \& Rangarajan, L., 2010. Face Identification from Manipulated Facial Images Using SIFT. 2010 3rd International Conference on Emerging Trends in Engineering and Technology, pp.192-195. Available at: http://ieeexplore.ieee.org/lpdocs/epic03/wrapper.htm?arnum ber $=5698318$.

15. Lu, X.L.X., Wang, Y.W.Y. \& Jain, A.K., 2003. Combining classifiers for face recognition. 2003 International Conference on Multimedia and Expo., 3, pp.13-16.

16. Mukhedkar, M.M., 2015. Fast Face Recognition Based on Wavelet Transform on PCA. , (Icesa), pp.761-764.

17. Tian, Y. et al., 2003. Do singular values contain adequate information for face recognition? Pattern Recognition, 36(3), pp.649-655.

18. Yang, J. et al., 2004. Two-Dimensional PCA: A New Approach to Appearance-Based Face Representation and Recognition. IEEE Transactions on Pattern Analysis and Machine Intelligence, 26(1), pp.131-137. 